\title{
Perforated Diverticulum of the Proximal Jejunum with
}

\begin{abstract}
Abscess
Jurij Janež ${ }^{*}$ and Klemen Mihelčič ${ }^{2}$

${ }^{1}$ Department of Abdominal Surgery, University Medical Centre Ljubljana, Slovenia

${ }^{2}$ University of Ljubljana, Medical Faculty, Slovenia

*Corresponding author: Jurij Janež, Department of Abdominal Surgery, University
\end{abstract}

Medical Centre Ljubljana, Zaloška Cesta 7, 1525 Ljubljana, Slovenia, Tel: +386 51315 815; E-mail: jurij.janez@gmail.com

\section{Abstract}

We present a case of an older male patient, who was referred to our department due to long lasting abdominal pain. He had undergone abdominal X-ray, US and CT with contrast but the underlying cause could not have been unequivocally determined. The patient underwent an explorative laparotomy during which an abscess as a consequence of perforated jejunal diverticulum has been found and successfully evacuated.

Keywords: Jejunum; Diverticulum; Perforation; Surgery; Acquired Entity

\section{Introduction}

Jejunal diverticula are a rare, acquired entity. They are usually asymptomatic [1-7]. When symptomatic they present with chronic non specific symptoms like pain, nausea and malnutrition but they may also present as an acute abdominal emergency. Complications include gastrointestinal haemorrhage, intestinal obstruction and perforation [5,7]. Chronic pain and malabsorption occur in $40 \%$ of cases while perforation occurs in only $2.3-6.4 \%$ of patients [7]. Due to its rarity, diagnosis is often late which consequently results in high morbidity and mortality associated with this disease [4].

We present a case of perforated diverticulum of the proximal jejunum with abscess diagnosed during explorative laparotomy.

\section{Case Report}

In this case report we present a case of a 79-year-old male patient, who was referred to our department due to pain in the abdomen lasting for 14 days with the pain increasing in severity the past few days. The patient has not vomited nor he felt nauseous. Miction and defecation were normal. His blood pressure at admission was $96 / 66$ $\mathrm{mmHg}$, body temperature $37,8^{\circ} \mathrm{C}$ and heart rate $88 / \mathrm{min}$. On the left side periumbilically abdomen was painful to palpation and rigid. Abnormal laboratory values included elevated Glu 6,8 mmol/L, Leu 10,1 mmol/L, CRP $93 \mathrm{mg} / \mathrm{L}$, total Bilirubin $20 \mu \mathrm{mol} / \mathrm{L}$, direct Bilirubin $7 \mu \mathrm{mol} / \mathrm{L}$, gama-GT 2,48 $\mu \mathrm{kat} / \mathrm{L}$ and macrocytic anemia (lowered RBC 3,06 x 10^12/L, Hb 104 g/L, Ht 29\%, MCH 34,1 pg, MCHC $358 \mathrm{~g} / \mathrm{L}$ ). His PT and INR were 0,21 and 3,12 respectively (due to being on a therapy due to atrial fibrillation). 15 years ago he had surgery due to umbilical hernia. 9 months after, he suffered abdominal trauma for which he needed surgery during which a lot of his bowel had been removed.

The abdominal X-ray showed no signs of free air in the abdominal cavity (Figures $1 \& 2$ ) while the chest X-ray showed signs of emerging pleural effusion on the left side (Figure 3). 


\section{Medical Journal of Clinical Trials \& Case Studies}
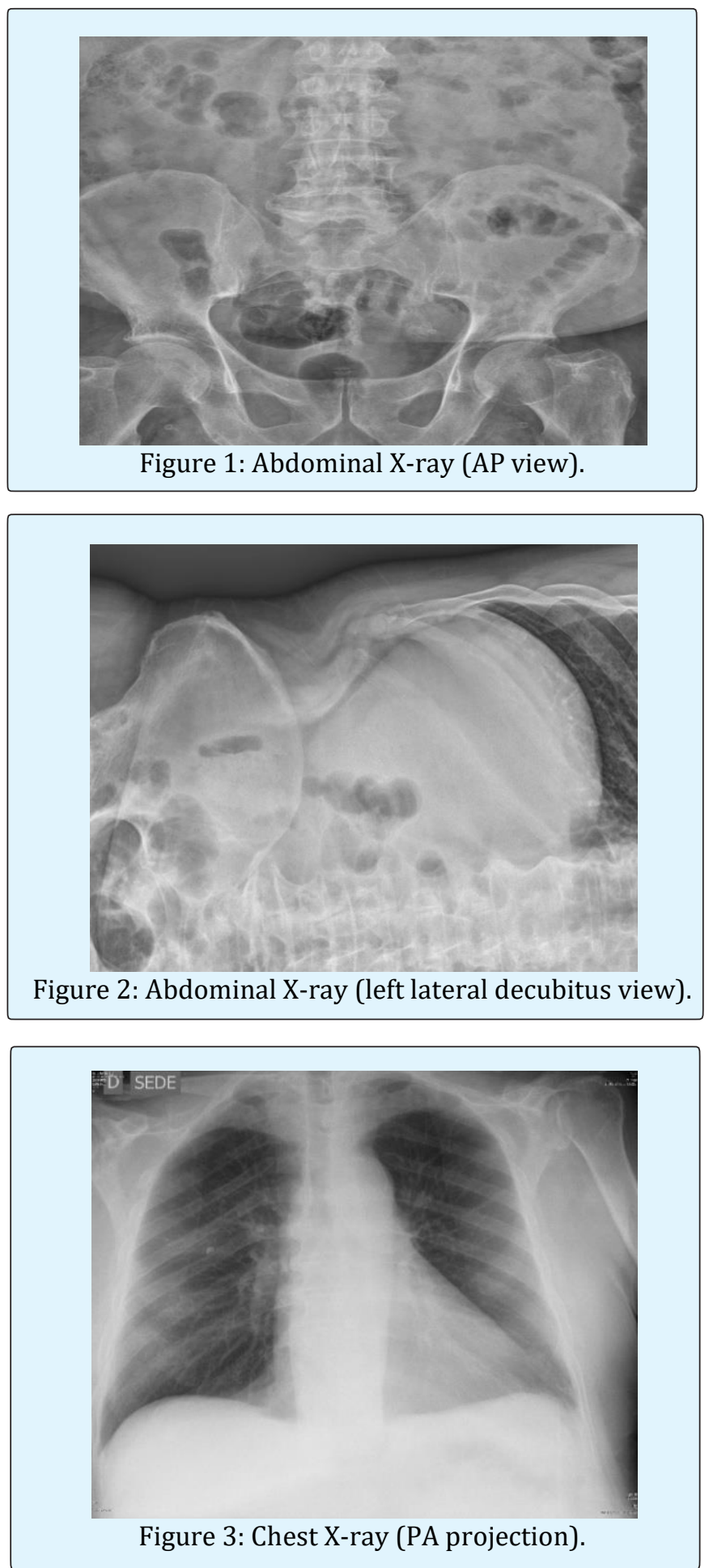

Abdominal ultrasound showed nothing that would explain patient's clinical picture. It only showed an extensive bowel meteorism and liver steatosis and hemangioma. CT with contrast confirmed the liver hemangioma and spoke of inguinal hernia that was not causing any obstruction. Diverticula of colon and sigma were present. There was no air or fluid intraperitoneally (Figure 4).

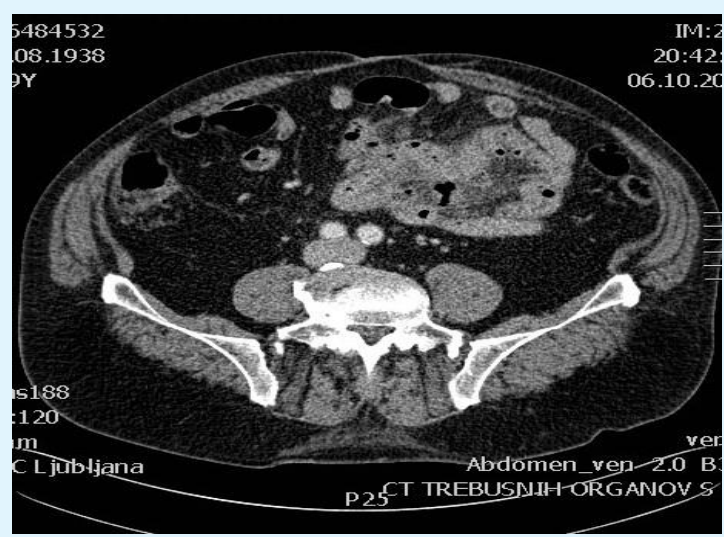

Figure 4: CT of the abdomen with contrast (transverse view).

The patient was sent to the operating theater. He was placed in a supine position and endotracheally intubated. The operative field was prepared in a sterile manner. An explorative laparotomy was performed. An abscess as a consequence of perforated divertuculum of the jejunum had been discovered and evacuated. A sample of the abscess had been taken and a drain had been inserted into the abdominal cavity. An adhesiolysis of a few glued small bowel loops had also been performed. The laparotomy was sutured with interrupted resorbable sutures and the skin wound was closed with staples.

Postoperative control CT of the abdomen confirmed that there were no signs of any abscess in the abdominal cavity but the diverticula of the jejunum were present. There were also some gas bubbles in the mesenteric fat that were suspicious for perforation.

The patient needed a prolonged antibiotic therapy after the surgery due to the elevated inflammatory parameters that have been slowly decreasing. He had a prolonged postoperative bowel paresis that was managed conservatively with nasogastric suction, prokinetic agents and bowel enemas. The other postoperative course was uneventful.

\section{Discussion}

Jejunal diverticula are outpouching of the intestinal wall. They fall in the category of false diverticula because they lack a muscular wall [2]. $75 \%$ are found in the proximal jejunum, $20 \%$ in the distal jejunum and $5 \%$ in the ileum [2,6]. They usually arise along with other 


\section{Medical Journal of Clinical Trials \& Case Studies}

diverticulae elsewhere in the abdomen such as the colon in up to $75 \%(30-75 \%)$ of cases which may suggest a potential connective tissue disorder [6,7]. They are rarely solitary(1). Their size varies between a few millimeters to greater than ten centimeters [2]. They are most frequently encountered in the elderly with slight male predominance $[2,5]$.

The true etiology has not yet been fully established but is likely multifactorial. It is postulated that they develop as a consequence of peristaltic dyskinesia which in turn leads to high intraluminal pressure which causes herniation of the mucosa and submucosa. This occurs on the antimesenteric border [3].

During the diverticular process stasis and bacterial overgrowth are prominent hence hyperbilirubinaemia can occur both via malabsorption and by bacterial mediated deconjugation. Bacteria also release siderophores and up regulate hepcidin, which can cause anaemia [3].

Jejunal diverticula are nearly 18 times more likely to perforate than duodenal diverticula [3]. Perforation may present as a localized perforation with or without generalized peritonitis. Alternatively, abscess can form as a consequence of perforation. Perforation carries an overall mortality rate of up to $40 \%$ [6].

Computed tomography is the most useful diagnostic imaging modality in diagnosing jejunal diverticula most often demonstrating jejunal thickening, inflammation or localized abscess formation [1,3,5]. Nevertheless currently, direct visualisation of diverticula via explorative laparoscopy or laparotomy is the only way of confirmation.

Non-operative management of a perforated jejunal diverticulum can be performed by an antibiotics course with or without CT-guided drainage of the abscess in stable patients presenting with localized abdominal symptoms and signs, but surgical exploration, lavage of the abdominal cavity and segmental resection with primary anastomosis remains the primary management of this disease in order to avoid sepsis [3]. Extensive resection should be avoided if possible as it has the potential to lead to short bowel syndrome $[3,5,6]$. Other procedures such as simple closure and excision are associated with an approximately three times greater mortality rate [7].

\section{Conclusion}

Jejunal diverticulosis is a rare pathologic entity, usually with late diagnosis in turn meaning it is associated with high morbidity and mortality. If it is symptomatic it can present itself with chronic non specific symptoms such as pain, nausea and malnutrition or as an acute emergency with complications such as perforation, GI bleeding and intestinal obstruction that require surgical intervention. It is important to keep in mind that a hyperbilirubinemia and anemia in a patient with chronic non specific abdominal symptoms raise a significant suspicion of jejunal diverticulosis. Best imaging modality for its discovery is abdominal CT but the best option overall is still a diagnostic explorative laparoscopy or laparotomy. In a stable patient with localized symptoms and signs the antibiotic course with drainage of the abscess can be performed but a surgery with segmental resection and an anastomosis is the recommended treatment of choice.

\section{References}

1. Baksi A, Gupta S, Kumar S, Ray U (2014) Perforated isolated jejunal diverticulum: a rare aetiology of acute abdomen. BMJ Case Reports.

2. Hubbard TJE, Balasubramanian R, Smith JJ (2015) Jejunal diverticulum enterolith causing perforation and upper abdominal peritonitis. BMJ Case Reports.

3. Kavanagh C, Kaoutzanis C, Spoor K, Friedman PF (2014) Perforated jejunal diverticulum: a rare presentation of acute abdomen. BMJ Case Reports.

4. Lempinen M, Salmela K, Kemppainen E (2004) Jejunal diverticulosis: a potentially dangerous entity. Scandinavian Journal of Gastroenterology 39(9): 905909.

5. Patel VA, Jefferis $H$, Spiegelberg $B$, Iqbal $Q$, Prabhudesai A, et al. (2008) Jejunal diverticulosis is not always a silent spectator: A report of 4 cases and review of the literature. World Journal of Gastroenterology 14(38): 5916-5919.

6. Sehgal R, Cheung CX, Hills T, Waris A, Healy D, et al. (2016) Khan T. Perforated jejunal diverticulum: a rare case of acute abdomen. Journal of Surgical Case Reports 2016(10): rjw169.

7. Singh S, Sandhu HPS, Aggarwal V (2011) Perforated Jejunal Diverticulum: A Rare Complication. Saudi Journal of Gastroenterology 17(5): 367. 